\title{
Physical Activity for Adults with Visual Impairments: Impact of Socio- Demographic Factors
}

\author{
Justin A. Haegele ${ }^{1}$, Xihe Zhu ${ }^{1}$, Jihyun Lee ${ }^{2}$, \& Lauren J. Lieberman ${ }^{3}$ \\ ${ }^{1}$ Old Dominion University, ${ }^{2}$ San Francisco State University, ${ }^{3}$ The College at Brockport, State University \\ of New York
}

Little is known about what factors influence physical activity participation for adult-aged individuals with visual impairments. Therefore, the purpose of this study was to explore the impact of sociodemographic factors on the physical activity participation of adults with visual impairments. The international physical activity questionnaire-short form and a socio-demographic factor questionnaire were distributed to individuals with visual impairments. A total of 176 adults $\left(M_{\text {age }}=47.04,52.8 \%\right.$ female) completed the questionnaires. Physical activity and socio-demographic variables were analyzed descriptively and relationships were explored using correlation analysis and multiple regression analysis. On average, participants reported 413.79 minutes of moderate-to-vigorous physical activity and 2058.52 minutes of sedentary active per week. A significant amount of variance of physical activity was explained by the socio-demographic variables in this study. Of the socio-demographic variables measured, only gender emerges as a significant positive predictor of total weekly physical activity. The results of this study both affirm and conflict with previous research. Unlike previous research focusing on those with visual impairments, this study demonstrated that gender was an important predictor of physical activity. This finding is consistent with population-wide data on individuals without disabilities in the United States.

Keywords: Gender, Disability, IPAQ, Health, Visual Impairment, Blindness

Currently, more than half of all deaths in the United States (US) are due to chronic, noncommunicable, lifestyle-mediated diseases (Booth, Roberts, \& Laye, 2012; Cardinal, Kang, Farnsworth II, \& Welk, 2015). It has been well documented that regular engagement in physical activity can help to improve the overall health and fitness, and reduce the risk for many of these chronic diseases (Centers for Disease Control \& Prevention [CDC], 2014; Kahn et al. 2002; Sallis, Prochaska, \& Taylor, 2000). In general, those who regularly engage in physical activity live longer and have a lower risk for diseases such as heart disease, stroke, depression, and obesity (CDC, 2014). Because of the importance of a physically active lifestyle, the United States Department of Health and Human Services (USDHHS; 2008) has established recommendations for American adults and youth to gain substantial healthrelated benefits of physical activity. The USDHHS (2008) suggests that adults participate in at least 150 minutes of moderate-intensity aerobic physical activity or 75 minutes of vigorous-intensity physical activity, in addition to muscle-strengthening activities at least two times per week. However, only $20 \%$ of adults in the US report engaging in recommended amounts of physical activity and musclestrengthening activities, with $25.4 \%$ report engaging in no leisure-time physical activity at all (CDC, 2014). With inadequate physical activity, individuals are at a higher risk for developing chronic diseases associated with inactivity, which can also lead to higher health 
care-related expenditures (Carlson, Fulton, Pratt, Yang, \& Adams, 2015).

Physical inactivity is particularly prevalent among adults with disabilities (Rimmer, 2008; Rimmer, Riley, Wang \& Rauworth, 2004; Rimmer \& Rowland, 2008). Nearly half of all adults with disabilities report being physically inactive and those that are inactive are 50\% more likely to report experiencing a chronic disease than those who were active (Carroll et al., 2014). Of those with disabilities, individuals with visual impairments, including those with low vision as well as those with complete blindness, tend to be among the least physically active (Longmuir \& Bar-Or, 2000). Recent research suggests that adults (Marmeleira, Laranjo, Marques, \& Pereira, 2014; Sadowska \& Krzepota, 2015) and youth (Aslan, Calik, \& Kitis, 2012; Haegele \& Porretta, 2015) with visual impairments tend to participate in inadequate amounts of physical activity. Conversely, those with visual impairments tend to spend the majority of their leisure-time in sedentary activities, such as watching television or playing computer games (Holbrook, Caputo, Perry, Fuller, \& Morgan, 2009; Marmeleira et al., 2014). Because of inadequate physical activity participation and a preference for sedentary behaviors, individuals with visual impairments are at higher risk for developing lifestyle mediated and health-related conditions, such as obesity (Holbrook et al., 2009; Ray, Horvat, Williams, \& Blasch, 2007).

Considering the growing evidence suggesting the importance of physical activity throughout the lifespan, it is important to understand what factors influence physical activity engagement of adults with visual impairments (Thomas, Halbert, Mackintosh, Quinn, \& Crotty, 2012). For typically developing individuals, research suggests that physical activity tends to vary dependent on a number of socio-demographic factors (Carlson et al., 2015; CDC, 2014). For example, population-based research has found that the prevalence of physical activity tends to be higher among males, younger age groups, and non-Hispanic white individuals (Carlson et al., 2015; CDC, 2014). Furthermore, those with more post-secondary education (e.g., higher education) and those whose family income is above the poverty level are more likely to meet physical activity recommendations (CDC, 2014). Unlike evidence pertaining to adults without disabilities, little is known about which factors influence physical activity participation for adult-aged individuals with visual impairments, and most research in this arena has been conducted focusing on school-aged individuals (Capella-Mcdonnall, 2007). Furthermore, preliminary research focusing on adults suggests that a number of sociodemographic factors that influence physical activity for typically developing individuals, such as income level, gender, and body-mass index, may not influence physical activity for those with visual impairments (Jaarsma, Dekker, Koopsman, Dijstra \& Geerstzen, 2014; Marmeleira et al. 2014). Other sociodemographic factors specific to those with visual impairments, such as visual impairment classification level and whether the impairments are congenital or acquired (e.g., on-set later in life), have also been found not to influence physical activity participation (Marmeleira et al. 2014). However, these factors may influence the types of activities that those with visual impairments choose (Lieberman \& McHugh, 2001) and barriers one may experience when participating in activities (Jaarsma et al,. 2014). While more substantial, research focusing on physical activity for school-aged individuals with visual impairments provides conflicting evidence regarding the influence of these sociodemographic factors. Specifically, conflicting evidence exists pertaining to the influence of visual impairment classification, age, and gender of participants and their physical activity participation (Aslan et al., 2012; Augestad \& Jiang, 2015; Ayvazoglu, Oh, \& Kozub, 2006; Houwen, Visscher, Hartman, Lemmick, 2007; Kozub \& Oh, 2006; Longmuir \& Bar-Or 2000). 
Therefore, the purpose of this study was to explore the impact of socio-demographic factors on the physical activity participation of adults with visual impairments.

\section{Participants}

\section{Methods}

Participants in this study were adults (aged 18 years or older) with visual impairments. A total of 176 individuals with visual impairments successfully completed the survey. Only completed surveys were included in the study. The participants age ranged from 22-77 years (mean age 47.04 years; $S D=13.18$ ), and $52.8 \%$ were female and $47.2 \%$ were male. Onset of visual impairment was almost equal among participants $(50.6 \%$ congenital and $49.4 \%$ after birth) and most participants reported having a B1 level visual impairment (i.e., blind; 53.4\%), with $30.7 \%, 12.5 \%$, and $3.4 \%$ reporting B3 (i.e., low vision), B2 (i.e., travel vision), and B4 (i.e., legal blindness) visual impairment levels, respectively. Among the participants, 9.7\% identified as being African American, 4.5\% as Asian American, 76.7\% as Caucasian, 5.1\% as Hispanic, and 3.9\% as other. Regarding K-12 education, most participants $(82.4 \%)$ attended public schools, while $2.8 \%$ attended a school for the blind and $1.1 \%$ of the participants were homeschooled. In addition, $13.6 \%$ of participants reported attending a combination of schools.

Table 1. Predictor category and frequency

\begin{tabular}{|c|c|c|c|c|c|}
\hline Predictor & Category1/\% & Category $2 / \%$ & Category3/\% & Category $4 / \%$ & Category $5 / \%$ \\
\hline Gender & Female/52.8 & Male/47.2 & & & \\
\hline Ethnicity/race & African Am/9.7 & Caucasian/76.7 & Asian Am/4.5 & Hispanics/5.1 & Other/3.9 \\
\hline VI classification & $\mathrm{B} 1 / 53.4$ & $\mathrm{~B} 2 / 12.5$ & B3/30.7 & B $4 / 3.4$ & \\
\hline Onset & Congenital/50.6 & After birth/49.4 & & & \\
\hline K-12 education & Public School/82.4 & School for Blind/2.8 & Combo/13.6 & Homeschool/1.1 & \\
\hline Mobility aid & Guide $\operatorname{dog} / 22.2$ & Cane/62.5 & None/13.6 & Wheelchair/1.7 & \\
\hline College education & $\mathrm{No} / 12.5$ & Some/28.4 & Bachelor/31.8 & Advanced/27.3 & \\
\hline Physical activity & Low/28.4 & Moderate/28.4 & High/43.2 & & \\
\hline
\end{tabular}

Note. VI = Visual impairment.

Table 1 provides further information regarding the percentage of the study population in regard to each of the measured sociodemographic factors (e.g., ethnicity/race, visual impairment classification, K-12 education).

\section{Data Collection}

The two questionnaires (of total of 16 questions) were distributed to participants utilizing the online, Google Drive platform. This online platform, and the uploaded survey, was checked for compatibility and accessibility using computer software commonly used by those with visual impairments by an accessibility expert at a school for the blind prior to distribution. All recommended modifications to the instrument were made.

Two visual impairment-related organizations in the United States (US) agreed to distribute access to the questionnaires. The two organizations utilized different procedures, based on each organization's code of conduct, to distribute the questionnaires. The first organization was composed of 14 small (e.g., approximately 12 members) groups of individuals (i.e., teams). This organization distributed participant recruitment information to one point person from each group (i.e., the team captain), with directions for them to share the information with the rest of the group. This organization sent out an introductory email, 
along with two email remainders. The second organization is a participant registry which is housed at a research university in the southern US. This registry is composed of adults with visual impairments who have interest in participating in research, and maintains a policy to allow external researchers to utilize the research registry to contact potential study participants. This organization distributed study information directly to each potential participant, and agreed to send one reminder email to the potential participants. Because of the nature of the two organizations, it is unknown how many individuals with visual impairments, in total, received the invitation to participate in the study. View (i.e., ratio of unique survey visitors/ unique site visitors), participant (i.e., ratio of unique visitors who agreed to participate/ unique first survey page visitors), and completion rates (i.e., ratio of users who finished the survey/ users who agreed to participate of the online survey) were not computed.

Participant recruitment information was distributed to potential participants through email. A cover letter in the email explained the purpose, methodology, and projected time commitment of the study. Inclusion criteria for this study, as stated in the cover letter, included that potential participants (a) were 18 years or older and (b) self-reported having a visual impairment. This letter assured participants that all data would be processed anonymously and that participation was voluntary. Those who volunteered were invited to click a link to proceed to the online questionnaire. Participants $(n=3)$ who were unable to complete the survey utilizing the online platform were encouraged to contact the first author, and complete the survey via telephone interview. All data were inputted, by the online platform or first author, into a savable and storable excel spreadsheet for data analysis. This survey was voluntary in nature and no incentives were offered in exchange for participation. The procedures for this study were approved by the institutional review board
(IRB) at the lead author's university of affiliation.

\section{Physical activity}

\section{Measures}

The international physical activity questionnaire-short form (IPAQ-SF) was selected to measure self-reported physical activity and sedentary behavior in this study (Craig et al., 2002). The IPAQ-SF is a sevenday recall instrument that asks participants to report time spent for various physical intensities: (a) walking, (b) moderate physical activity, (c) vigorous physical activity, and (d) sitting (i.e., sedentary behavior). Participants are asked to refer to both planned (e.g., exercise, recreation) and unplanned (e.g., transportation, housework) physical activity. Craig and colleagues (2002) tested the IPAQ-SF for reliability $(\rho=0.76)$, criterion validity $(\rho=$ $0.30)$, and concurrent validity $(\rho=0.67)$ and values are considered acceptable for adults who are sighted. Recently, the IPAQ-SF demonstrated moderate and acceptable levels of criterion validity and user sensitivity for use with adults with visual impairments and has demonstrated moderate correlations with objective measures (from $r=0.38$ to $r=0.57$; Marmeleira, Laranjo, Marques, \& Batalha, 2013; Sadowska \& Krzepota, 2015). The SF version of the IPAQ was selected for this study, as opposed to the long form, because validation information regarding the utilization of the long form for individuals with visual impairments is currently unavailable.

\section{Socio-demographic factors}

A questionnaire was utilized to gather participant socio-demographic factors. This questionnaire included nine items, and asked participants to report (a) age, (b) gender, (c) ethnicity/ race, (d) college education, (e) visual impairment classification, (f) K-12 education, and (g) whether participants used a mobility 
device (e.g., long cane) and if so, which one. Furthermore, participants were asked whether their visual impairment was congenital (at birth) or after birth, and those who indicated that their impairment occurred after birth were asked to answer a subsequent question asking how many years they have had a visual impairment. Visual impairment classification was based on the United States Association of Blind Athletes (2013) visual impairment classification system, and participants had the option to select B1, B2, B3 and B4. Regarding K-12 education, participants were asked whether they attended a residential school for the blind, a public school, a combination, or were home-schooled.

\section{Data Analysis}

First, the IPAQ-SF variables, including weekly moderate-to-vigorous physical activity (MVPA), sedentary time, and total metabolic (MET) minutes, and socio-demographic variables, were descriptively analyzed. More specifically, descriptive statistics involved frequency, central tendencies, and standard deviation data. Weekly MET minutes were derived utilizing a standardized IPAQ data conversion protocol (IPAQ, 2005). Conversion procedures utilized the standard MET-min/week formula (minutes-per-day $\mathrm{x}$ days-per-week $\mathrm{x}$ MET intensity) and standard MET intensities for walking (3.3 METs), moderate-physical activity (4 METs), and vigorous physical activity (8 METs). One MET is considered equal to the energy expenditure while resting. Total MET-min/week represented a weighted estimate of total physical activity across all three categories (e.g., vigorous, moderate, and walking).

In order to examine relationships among the quantifiable variables, a correlation analysis was conducted among age, years of visual impairment, and IPAQ-SF variables. To examine the impacts of the socio-demographic variables on self-reported physical activity participation of the adults with visual impairments, a multiple regression analysis was conducted with total MET minutes per week as the dependable variable, and the sociodemographic variables as independent variables. Throughout the analyses, alpha value was set at 0.05 .

\section{Results}

Table 2. Descriptive Results of the Variables

\begin{tabular}{lllll}
\hline Variables & $M$ & $S D$ & Min. & Max. \\
\hline Age & 47.04 & 13.21 & 18.00 & 77.00 \\
Years & 37.49 & 17.18 & 1.00 & 77.00 \\
Weekly MVPA Min/Wk & 413.79 & 636.79 & 0.00 & 3600.00 \\
Weekly Sedentary Min/Wk & 2058.52 & 1690.56 & 35.00 & 9660.00 \\
Weekly Total MET Min/Wk & 2845.55 & 4504.51 & 0.00 & 26340.00 \\
\hline
\end{tabular}

Note. $\mathrm{VI}=$ Visual impairment; $M=$ mean; $S D=$ standard deviation;

MVPA = moderate-to-vigorous physical activity;

$\mathrm{Min} / \mathrm{Wk}=$ Minutes per week.

As shown in Table 2, on average the participants reported 413.79 minutes of MVPA, $S D=636.79,2058.52$ minutes sedentary, $S D=$
1690.56, and total MET minutes of 2845.55, SD

$=4504.51$, per week. The correlation analysis results are presented in Table 3. 
Table 3. The Pearson Product-Moment Correlation Coefficients between Variables

\begin{tabular}{|c|c|c|c|c|c|}
\hline Variables & 1 & 2 & 3 & 4 & 5 \\
\hline 1. Age & 1 & & & & \\
\hline 2. Years $_{\mathrm{VI}}$ & $0.56^{*}$ & & & & \\
\hline 3. MVPA & -0.07 & -0.01 & & & \\
\hline 4. Sedentary & 0.04 & -0.07 & $-0.21^{*}$ & & \\
\hline 5. Total MET Min & -0.12 & -0.07 & $0.87^{*}$ & $-0.18^{*}$ & 1 \\
\hline
\end{tabular}

As expected, there was a significant positive correlation between age and years of visual impairment $(r=0.56, p<0.05)$, and between weekly MVPA time and weekly total MET minutes $(r=0.87, p<0.05)$. However, there was no significant correlation between age/years of visual impairment and weekly
MVPA, sedentary time, or total weekly MET minutes $(p>0.05)$.

Finally, a multiple regression analysis was conducted to examine how much the sociodemographic variables (in Table 3) can predict the participants' total weekly MET minutes.

Table 4. Multiple Regression Results

\begin{tabular}{llllll}
\hline Predictors & \multicolumn{5}{l}{$\begin{array}{l}\text { DV: Total MET min per week } \\
\end{array}$} \\
\cline { 2 - 6 } & $B$ & $S E$ & $\beta$ & $t$ & $P$ \\
\hline Intercept & -860.93 & 2540.73 & & -0.34 & 0.74 \\
Gender & 2288.18 & 670.65 & 0.25 & 3.41 & 0.00 \\
Ethnicity & 242.63 & 354.71 & 0.05 & 0.68 & 0.49 \\
VI Type & 365.85 & 382.36 & 0.08 & 0.96 & 0.34 \\
Onset & -209.77 & 713.72 & -0.02 & -0.29 & 0.77 \\
Years & -6.98 & 21.20 & -0.03 & -0.33 & 0.74 \\
K-12 Education & 94.72 & 448.43 & 0.02 & 0.21 & 0.83 \\
Mobility Aid & 618.93 & 565.20 & 0.09 & 1.09 & 0.27 \\
College Education & -582.58 & 309.71 & -0.14 & -1.88 & 0.06 \\
\hline Note. VI = visual impairment, SE $=$ standard error, $* p<0.05$. & &
\end{tabular}

As shown in Table 4, the regression results show that a significant amount of variance of the participants' total weekly MET minutes was explained by the socio-demographic variables $\left(F_{8,167}=2.76, p<0.05\right)$. Overall, the model explains about $11.66 \%$ of the variance in the participants' total weekly MET minutes. According to Cohen (1988), this result $\left(f^{2}=\right.$ 0.13 ) indicates an effect size close to the moderate threshold $\left(f^{2}=0.15\right)$. Gender was the only variable that emerged as a significant positive predictor for total weekly MET minutes $(\beta=0.25, p<0.05)$, while controlling for other factors. On average, males reported 572 more total weekly MET minutes than females when other factors were held constant.

\section{Discussion}

The purpose of this study was to explore the impact of socio-demographic factors on physical activity participation of adults with visual impairments. While research pertaining to adults without disabilities has demonstrated that age and ethnicity/race tend to impact physical activity, these variables have not been previously tested among adults with visual impairments. Unlike ethnicity/race, previous 
research suggests that age may influence physical activity among youth with visual impairments, demonstrating that older individuals with visual impairments tend to be less physically active than younger individuals (Haegele \& Porretta, 2015). The results of this study conflict with research pertaining to youth, and suggest that age is not a significant predictor of self-reported physical activity for adults with visual impairments. Similarly, unlike research pertaining to those without visual impairments, ethnicity/race was not found to impact physical activity participation. College education was another socio-demographic variable that was tested in this study, as previous research on adults without (CDC 2014) and with (Jaarsma et al, 2014) visual impairments indicates that those with postsecondary experience would be more physically active. However, in the current study, college education was not a significant predictor of physical activity. Interestingly, participants in this study (while not statistically significant negative $\beta$ value) with less college education experience reported more physical activity than those with more college education experience. These results conflict with research by Jaarsma et al (2014), but can be partly explained by use of a binary variable (i.e., having or not having post-secondary education experience) in their study and the use of different levels of experience in higher education (i.e., some bachelors experience, completed bachelors experience, etc.) in the current study.

This study explored the impact of a number of visual impairment specific sociodemographic factors on physical activity participation as well. Little previous research (Jaarsma et al., 2014; Marmeleira et al., 2014) has considered these variables in adults with visual impairments and the findings of those studies are consistent with the current research. Specifically, previous studies and the current study agree in that items such as the degree (e.g., B1, B2) and age of acquisition (e.g., congenital, after birth) of visual impairments do not predict physical activity participation (Jaarsma et al., 2014; Marmeleira et al., 2014). Interestingly, one study, though, found that adults with bilateral vision loss (i.e., vision loss in both eyes) tend to be less physically active than those with unilateral vision loss (i.e., vision loss in one eye; Van Landinham, Willis, Vitale \& Ramulu, 2012). However, the degree of visual impairment was the focus of the current study, which is not directly comparable to these findings. Research pertaining to youth with visual impairments that has explored the impact of degree of visual impairment on physical activity participation is conflicting. While evidence exists that is consistent with the current research (Kozub \& Oh, 2006), other previous research has demonstrated that youth with less vision (i.e., those with a more severe visual impairment) tended to be less physically active (Aslan et al., 2012; Houwen et al., 2007).

The current study is the first to explore the impact of K-12 education (e.g., public school, school for the blind, combination, homeschooled) on physical activity for individuals with visual impairments. We suspected that K12 education may have had an impact on physical activity participation, because students with visual impairments who are educated in residential schools for the blind are offered a variety of adapted activities designed to meet their specific needs. Furthermore, while public schools physical activity experiences for those with visual impairments have garnered much attention recently (Lieberman, Ponchillia, \& Ponchillia, 2013), students with disabilities tend to report a number of challenges in these settings such as social isolation and inability to participate in activities (Haegele \& Sutherland 2015). Contrary to our expectations, there were no significant differences in physical activity participation between adults with visual impairments who were educated in residential schools for the blind and public schools. While this may be an indicator of improvements in inclusive/public school physical education, it may also be a result of the lack of impact that 
school-based physical education programming has on adult physical activity behaviors.

Of the socio-demographic variables tested in this study, only gender was a significant predictor of self-reported physical activity participation among adults with visual impairments. This is the first study to discover gender differences among adults with visual impairments regarding physical activity participation, and conflicts with previous research utilizing both objective measures (Marmeleira et al., 2014) and self-report (Jaarsma et al., 2014). It is important to note that these comparable studies were both conducted outside of the US (i.e., Portugal, The Netherlands) and the contextual/cultural differences within the US may influence these gender discrepancies. Interestingly, the impact of gender on physical activity, namely males being significantly more physically active than females, is consistent with population-level research in the US focusing on adults without disabilities (Carlson et al., 2015; CDC, 2014).

The factors explored in this study explained approximately $11.66 \%$ of the variance in the participants' self-reported physical activity behavior. Although practically and statistically significant, this represents just a nominal portion of what may influence an individual with visual impairments to be physically active. To further explore physical activity behavior of this population, further research is necessary. Researchers may want to explore the impact of additional socio-demographic factors, such as socioeconomic status, that tend to impact physical activity participation of adults without disabilities in the US (CDC, 2014). A number of barriers to physical activity participation, such as lack of opportunities to participate and transportation issues, which are commonly expressed by those with visual impairments (Jaarsma et al., 2014; Phoenix, Griffin, \& Smith, 2015) can be associated with available funds one can allocate for physical activity. Because of this, those with visual impairments who are more financially comfortable may have a greater ability to access physical activity options than those who may not have those financial advantages.

In addition to socio-demographic factors that may influence physical activity, additional barriers and facilitators to physical activity participation must also be explored. Recently, Jaarsma and colleagues (2014) examined barriers to and facilitators of sports and physical activity participation among people with visual impairments in the Netherlands. Among participants who considered themselves active $(n=411)$, two commonly expressed barriers to being physically active were dependence of others to exercise and not being comfortable in the presence of other people. Furthermore, environmental barriers, such as transportation issues, close proximity opportunities, and insufficient facilities were commonly expressed (Jaarsma et al., 2014). Many of these barriers and facilitators are likely also relevant for those with visual impairments residing in other countries, however it is important to explore which barriers and facilities are most prominent in each context.

Although many participants in the current study report being physically active and exceeding the 150 minutes of moderate physical activity and/or 75 minutes of vigorous physical activity suggested by week by the USDHHS, weekly minutes of sedentary activity were also very high ( $M=2058$ minutes per week). Recent research suggests that even at times when adults do meet physical activity guidelines, participation in high volumes of sedentary behavior can compromise health (Owens, Healy, Matthews, \& Dunstan, 2010). Sedentary behaviors, such as sitting, can have undesirable health consequences such as increasing the risk of developing health-related issues (e.g., cardiovascular disease; Lynch, Dunstan, Vallance, \& Owens 2013; Starkoff et al., 2014). Because of this, it is essential for researchers to examine potential interventions to further enhance physical activity and decrease sedentary behaviors for this population. 
However, interventions focusing on this population are rare (Haegele \& Porretta, 2014; Skelton et al., 2013) and most previous intervention research in this area focuses on school-aged populations (Furtado, AllumsFeatherston, Lieberman, \& Gutierrrez, 2014).

\section{Limitations of the Study}

Certain limitations were evident in this study. First, the utilization of a self-report instrument to measure physical activity rather than an objective physical activity monitor may be viewed as a limitation. However, there are several reasons that went into the decision to utilize the self-report instrument. Namely, utilizing the self-report instrument allowed the researchers to gather data from a large number of participants in an economically efficient manner. Furthermore, although objective measures have gained popularity, Haskell (2012) notes that self-report instruments should continue to be considered a useful and valuable research methodology. Second, data for this study were collected via online survey, which may be considered a limitation because of sampling and access concerns (Wright, 2006). However, online surveys were selected because of the unique access this strategy provided to a large number of individuals with visual impairments in an economical and accessible manner (Wright, 2006). Lastly, the participants of this study are limited to individuals who volunteered to participate in research and had the capability to access the online survey. Because of this, the results may not be representative of the entire population of adults with visual impairments.

\section{Perspective}

The purpose of this study was to explore the impact of socio-demographic factors on physical activity participation of adults with visual impairments. The results of the current study are the first focused specifically on socio- demographic factors for this population, and expand our understanding of physical activity for adults with visual impairments. Our findings suggest that, among the socio-demographic factors explored, only gender significantly predicted physical activity participation of adults with visual impairments. No association was found between other socio-demographic factors, such as chronological age, visual impairment level, or ethnicity/race. Because of the importance of physical activity for all individuals, it is essential for future research to explore additional factors (e.g., socioeconomic status) that may impact physical activity participation for those with visual impairments. Furthermore, as described by CapellaMcDonnall (2007), little has been done to address physical activity deficiencies of adults with visual impairments and it is essential for future researchers to continue to explore strategies to impact physical activity participation for this population.

\section{References}

Aslan, U., Calik, B., \& Kitis, A. (2012). The effect of gender and level of vision on the physical activity levels of children and adolescents with visual impairments. Research in Developmental Disabilities, 33, 1799-1804. doi:10.1016/j.ridd.2012.05.005

Augestad, L.B., \& Jiang, L. (2015). Physical activity, physical fitness, and body composition among children and young adults with visual impairments: A systematic review. British Journal of Visual Impairment, 33(3), 167-182.

Ayvazoglu, N., Oh, H., \& Kozub, F. (2006). Explaining physical activity in children with visual impairments: A family systems approach. Exceptional Children, 72(2), 235248.

Booth, F.W., Roberts, C.K., \& Laye, M.J. (2012). Lack of exercise is a major cause of chronic diseases. Comprehensive Physiology, 2, 1143-1211. 
Capella-McDonnall, M. (2007). The need for health promotion for adults who are visually impaired. Journal of Visual Impairment \& Blindness, 101(3), 133-145.

Cardinal, B.J., Kang, M., Farnsworth II, J.L., \& Welk, G.J. (2015). Historical context and current status of the intersection of physical activity and public health: Results of the 2015 American Kinesiology Association's Opportunities for Kinesiology survey. Kinesiology Review, 4, 329-345.

Carlson, S.A., Fulton, J.E., Pratt, M., Yang, Z., \& Adams, E.K. (2015). Inadequate physical activity and health care expenditures in the United States. Progress in Cardiovascular Disease, 57(4), 315-323.

Carroll, D.D., Courtney-Long, E.A., Stevens, A.C., Sloan, M.L., Lullo, C., Visser, S.N., Fox, M.H., Armour, B.S., Campbell, V.A., Brown, D.R. and Dorn, J.M. (2014). MMWR Morb Weekly Report, 63(18), 407418.

Centers for Disease Control and Prevention. State Indicator Report on Physical Activity, 2014. Atlanta, GA: U.S. Department of Health and Human Services, 2014.

Craig, C.L., Marshall, A.L., Sjostrom, M., Bauma, A.E., Booth, M.L., Ainsworth, B.E., Pratt, M., Ekelund, U., Yhgve, A., Sallis, J.F., \& Oja, P. (2003). International physical activity questionnaire: 12-country reliability and validity. Medicine \& Science in Sports \& Exercise, 35, 1381-1395.

Furtado, O.L.P.C., Allums-Featherston, K., Lieberman, L.J., \& Gutierrez, G.L. (2015). Physical activity interventions for children and youth with visual impairments. Adapted Physical Activity Quarterly, 25(2), 156-176. doi:10.1123/APAQ.2014-0164

Haegele, J.A., \& Porretta, D.L. (2015). Physical activity and school-aged individuals with visual impairments: A literature review. Adapted Physical Activity Quarterly, 32(2), 68-82. doi:10.1123/apaq.201-0110

Haegele, J.A. \& Sutherland, S. (2015). The perspective of students with disabilities toward physical education: A review of qualitative inquiry. Quest, 67(3), 255273.doi:10.1080/00336297.2015.105118

Haskell, W.L. (2012). Physical activity by selfreport: A brief history and future issues. Journal of Physical Activity \& Health, 9, S5-S10.

Holbrook, E.A., Caputo, J.L., Perry, T.L., Fuller, D.K., \& Morgan, D.W. (2009). Physical activity, body composition, and perceived quality of life of adults with visual impairments. Journal of Visual Impairment \& Blindness, 103(1), 17-29.

Houwen, S., Visscher, C., Hartman, E., \& Lemmink, K. (2007). Gross motor skills and sport participation of children with visual impairment. Research Quarterly for Exercise \& Sport, 78(1), 16-23.

International Physical Activity Questionnaire (2005). Guidelines for data processing and analysis of the international physical activity questionnaire (IPAQ). Retrieved from www.ipaq.ki.se.

Jaarsma, E.A., Dekker, R., Koopsman, S.A., Dijkstra, P.U., \& Geerstzen, J.H.B. (2014). Barriers to and facilitators of sports participation in people with visual impairments. Adapted Physical Activity Quarterly, 31(3), 240-264. doi:10.1123/apaq.2013-0119

Kahn, E.B., Ramsey, L.T., Brownson, R.C., Health, G.W., Howze, E.H., Powell, K.E., Stone, E.J., Rajab, M.W., \& Corso, P. (2002). The effectiveness of interventions to increase physical activity: A systematic review. American Journal of Preventive Medicine, 22(4), 73-107.

Kozub, F. \& Oh, H. (2004). An exploratory study of physical activity levels of children and adolescents with visual impairments. Clinical Kinesiology, 58(3), 1-7.

Lieberman, L.J., \& McHugh, E. (2001). Healthrelated fitness of children who are visually impaired. Journal of Visual Impairment \& Blindness, 95, 272-288. 
Lieberman, L.J., Ponchillia, P., \& Ponchillia, S. (2013). Physical education and sport for individuals who are visually impaired or deafblind. New York, NY: American Federation of the Blind.

Longmuir, P., \& Bar-Or, B. (2000). Factors influencing physical activity levels of youth with physical and sensory disabilities. Adapted Physical Activity Quarterly, 17(1), 40-53.

Lynch, S.M., Dunstan, D.W., Vallance, J.K., \& Owens, N. (2013). Don't take cancer sitting down: A new survivorship research agenda. Cancer, 119, 1928-1935.

Marmeleira, J., Laranjo, L., Marques, O., \& Batalha, N. (2013). Criterion-related validity of the short from of the international physical activity questionnaire in adults who are blind. Journal of Visual Impairment \& Blindness, 107(5), 375-382

Marmeleira, J., Laranjo, L., Marques, O., \& Pereira, C. (2014). Physical activity patterns in adults who are blind as assessed by accelerometry. Adapted Physical Activity Quarterly, 31(3), 283-296. doi:10.1123/apaq.2013-0039.

Owens, N., Healy, G.N., Matthews, C.E., \& Dunstan, D.W. (2010). Too much sitting: The population health science of sedentary behavior. Exercise \& Sport Sciences Review, 38(3), 105-113. 10.1097/JES.0b013e3181e373a2

Phoenix, C.M., Griffin, M., \& Smith, B. (2015). Physical activity among older people with sight loss: A qualitative research study to inform policy and practice. Public Health, 129(2), 124-130.

Ray, C.T., Horvat, M., Williams, M., \& Blasch, B.B. (2007). Clinical assessment of functional movement in adults with visual impairments. Journal of Visual Impairment \& Blindness, 101, 108-113.

Rimmer, J.H. (2002). Health promotion for individuals with disabilities. The need for a transitional model in service delivery.
Disability Management \& Health Outcomes, 10, 337-343.

Rimmer, J.H., Riley, B., Wang, E., Rauworth, A., \& Jurkowski, J. (2004). Physical activity participation among persons with disabilities. American Journal of Preventive Medicine, 26(5), 419-425.

Rimmer, J.H., \& Rowland, J.L. (2008). Physical activity in youth with disabilities: A critical need in an underserved population. Developmental Neurorehabilitation, 11, 141-148.

Sadowska, D., \& Krzepota, J. (2015). Assessment of physical activity of people with visual impairments and individuals who are sighted using the international physical activity questionnaire. Journal of Visual Impairment \& Blindness, 109(2), 119-129.

Sallis, J.F., Prochaska, J.J., \& Taylor, W.C. (2000). A review of correlates of physical activity of children and adolescents. Medicine \& Science in Sports \& Exercise, 32(5), 963-975.

Skelton, D., Howe, T., Ballinger, C., Neil, F., Palmer, S., \& Gray, L. (2013). Environmental and behavioral interventions for reducing physical activity limitation in community-dwelling visually impaired older people. Cochrane Database of Systematic Review, 6, 1-27.

Starkoff, B.E., Petosa, R.L., Balk, E.K., Eneli, I.U., Bonny, A.E., Hoffman, R.P., \& Devor, S.T. (2014). Sedentary and Physical Activity Habits of Obese Adolescents. American Journal of Health Education, 45(6), 335-341.

Thomas, S., Halbert, J., Mackintosh, S., Quinn, S., \& Crotty, M. (2012). Sociodemographic factors associated with self-reported exercise and physical activity behaviors and attitudes of south Australians: Results of a population-based survey. Journal of Aging \& Health, 24(2), 287-306.

United States Association of Blind Athletes 
(USABA; 2013). IBSA visual classifications. Retrieved March14, 2013, from

http://usaba.org/index.php/membership/visu alclassifications/visual classifications/

U.S. Department of Health and Human Services. (2008). Physical Activity Guidelines for Americans. In: U.S. Department of Health and Human Services, editor. Hyattsville, MD, 2008.

Van Landinham, S.W., Willis, J.R., Vitale, S., \& Ramulu, P.Y. (2012). Visual field loss and accelerometer-measured physical activity in the United States. Ophthalmology, 119, 2486-2496.

Wright, K.B. (2006). Researching internet-based populations: Advantages and disadvantages of online survey research, online questionnaire authoring software packages, and web survey services. Journal of Computer-Mediated Communication, 10(3), doi:10.1111/j.1083 6101.2005.tb00259.x

Email: jhaegele@odu.edu 